\title{
Edad materna y defectos del tubo neural: evidencia para un efecto mayor en espina bífida que anencefalia
}

\author{
Alexandre R. Vieira*1, Silvia Castillo Taucher². \\ Influence of maternal age on the risk for \\ neural tube defects, a meta analysis
}

Background: Recent evidence from birth order data suggest that maternal factors can differently influence anencephaly and spina bifida. Aim: To study the influence of maternal age on the risk for neural tube defects. Material and methods: A meta-analysis of published data on neural tube defects (NTDs) was carried out to determine whether there is an increased risk to have a child with NTDs for younger and older mothers and if this risk differs depending on the type of NTD. All data available with information regarding the frequency of live births and NTDs cases by maternal age (five- or ten-year intervals) were included in the analysis. Effect sizes calculations were performed. Results: The analysis supports the hypothesis that there is an increased risk of having an offspring with NIDs for mothers 40 years of age or older. However, this effect is stronger for spina bifida than for anencephaly. There is also evidence that mothers 19 years old or younger have a higher risk for having a child with spina bifida. Conclusions: Maternal age influences the risk of having an offspring with neural tube defects (Rev Méd Chile 2005; 133: 62-70).

(Key words: Anencephaly; Maternal age; Neural tube defects; Spinal dysraphism)

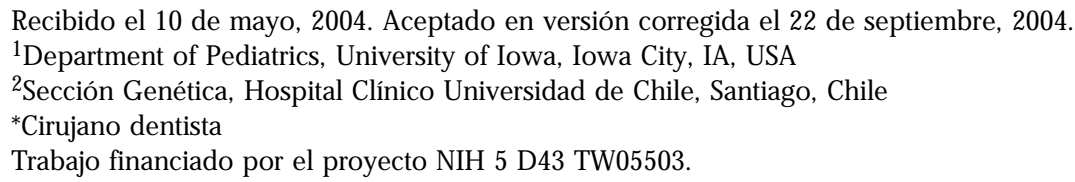

$E^{n}$ la sociedad actual, se ha hecho más común para las mujeres el considerar un embarazo a la edad de 35 o más años, por una variedad de razones. Algunas han privilegiado su educación, sus carreras, o se han casado más tardíamente de lo que solían las mujeres décadas atrás. Otras, simplemente, desean tener un hijo más en su vida, incluso después de uno o dos embarazos previos. Los problemas de infertilidad son más prevalentes a mayor edad materna, sin embargo, la tecnología ha contribuido a ayudar a muchas mujeres en lograr un muy ansiado embarazo. Estadísticamente, hay muchas más mujeres con edad sobre los 35 años que en cualquier otra era. Este fenómeno

Correspondencia a: Alexandre R. Vieira. Dept Pediatrics ML 2182, The University of Iowa. Iowa City, IA 52242 USA. Phone \# (319) 335-9686. FAX \# (319) 335-6970. E-Mail: alexandre-vieira@uiowa.edu implica un mayor número de mujeres en riesgo para anomalías cromosómicas como el síndrome de Down. Un número de otras enfermedades, incluyendo defectos del nacimiento, también han sido investigados por el impacto de una mayor edad materna. Los defectos congénitos son la primera causa de muerte en países desarrollados y la segunda en muchos países en vías de desarrollo. Las malformaciones congénitas aisladas son usualmente rasgos complejos. El conocimiento de un factor de riesgo para estos problemas sería un importante logro. Entre estos factores de riesgo, la edad maternal es uno de los más estudiados.

Los defectos del tubo neural (DTN) están entre los más comunes de las malformaciones congénitas más serias. Un DTN en el feto es aquél que afecta las estructuras que constituyen el cerebro y la columna vertebral. Los dos tipos más importan- 0 
tes de DTN son anencefalia, es decir, la ausencia parcial o completa del cerebro, con un daño muy extenso que determinará que el niño sea un mortinato o fallezca muy luego después del nacimiento, y la espina bífida, en que hay un cierre incompleto de la columna vertebral, que requiere cirugía para cubrir y prevenir daños subsecuentes. Estos niños pueden evolucionar con hidrocefalia, tener diversos grados de compromiso motor y sensitivo en sus extremidades inferiores y problemas de continencia urinaria y digestiva. Como secuelas pueden presentar trastornos de aprendizaje y, algunos, retraso mental.

Estos defectos ocurren muy temprano, generalmente en el primer mes de desarrollo embrionario, cuando aún el diagnóstico de embarazo puede ser incierto.

Hoy, la prevalencia de defectos del tubo neural es alrededor de 6 en cada 10.000 nacidos vivos en el mundo. Noventa por ciento de los niños con DTN nacen en familias donde esto nunca ha ocurrido antes. Setenta y cinco por ciento de los embarazos afectados termina en aborto o mortinato y sólo $25 \%$ de estos niños nace vivo. Se cree que tanto factores genéticos (heredados) como ambientales contribuyen en la etiología de DTN.

La literatura que trata sobre la posible relación entre edad materna y defectos del tubo neural ha sido considerada muy desorientadora. La variación más frecuentemente reportada, particularmente en literatura más antigua, ha sido una relación en forma de $U$ entre la prevalencia de defectos del tubo neural y edad materna, con tasas mayores en madres bajo 20 y en aquellas sobre 35 años, con una tasa mínima entre 20 y 24 ó 25 y 29 años de edad $^{1}$. Los efectos por edad materna son reducidos, y en la mayoría de los estudios que han sido analizados adecuadamente, son mucho menos marcados que los efectos de orden de nacimiento. Una revisión de datos de orden de nacimiento en defectos del tubo neural sugiere que un leve efecto de orden de nacimiento puede ser visto para espina bífida (tamaño del efecto $=0,35$ ), pero no para anencefalia, por ello, juntar datos de anencefalia y espina bífida pudiera ser una de las explicaciones para las controversias encontradas en la literatura ${ }^{2}$. El presente trabajo revisa datos publicados en relación a defectos del tubo neural y edad materna, para investigar si los datos combinados sólo para un tipo específico de defecto del tubo neural (por ejemplo, anencefalia o espina bífida), proveen una explicación para la asociación reportada entre defectos del tubo neural y edad materna.

\section{MÉTodo}

Se utilizaron dos enfoques básicos para obtener literatura, en cualquier idioma, relacionando edad materna y DTNs. Primero, se revisaron las bases de datos de Medline, Embase, y Science Citation Index, mediante un compendiador, usando las palabras clave «defectos del tubo neural», (anencefalia», sespina bífida» «defectos del sistema nervioso central», mielomeningocele», «edad matema» y sedad paterna», para estudios publicados desde 1966 hasta 2000. Se identificaron un total de 3.883 publicaciones. Se preseleccionaron todas las publicaciones que presentaban información en relación a edad matema en DTNs. Después, en cada uno de estos estudios obtenidos en la búsqueda de bases de datos, se revisaron las listas de referencias para seleccionar más referencias. Se seleccionaron un total de 118 publicaciones. Todos los estudios basados en las poblaciones con información relacionada al número de nacidos vivos y casos de DTNs por edad matema (intervalos de 5 ó 10 años) fueron incluidos en este estudio. La mayoría de los estudios caso-control no proveyeron controles adecuadamente pareados, en consecuencia, se excluyeron del análisis los estudios sin especificación de la frecuencia de casos o de nacimientos vivos por edad matema. Se seleccionaron treinta y tres publicaciones usando los criterios descritos (Tabla 1). Se pueden encontrar detalles en relación a la identificación de casos en los estudios individuales en las publicaciones originales.

En el presente estudio, la asociación entre grupos de edad materna y anencefalia, espina bífida, y DTNs fue medida separadamente en cada estudio por el odds ratio y su intervalo de confianza de 95\%. Las edades 25-29 ó 20-29 fueron consideradas como el grupo de referencia (odds ratio $=1,0$ ). Los tests exactos de Fisher fueron realizados, también, para obtener valores de ji-cuadrado, que fueron usados en los cálculos de los metaanálisis. Todos los análisis estadísticos fueron conducidos utilizando la version 6.03 de Epi Info ${ }^{3}$. Después, se utilizaron los tamaños de los efectos para realizar un metaanálisis ${ }^{4}$. Una primera aproximación fue la aplicación de un modelo de efecto fijo (Weighted 
Integration Method) ponderando los datos de acuerdo a los tamaños muestrales, ya que la varianza de un efecto simple depende de su tamaño muestral. El tamaño de los efectos basados en muestras mayores son más precisos $\mathrm{y}$, por ese motivo, merecen mayor peso. El supuesto subyacente era que los set de datos eran homogéneos, es decir, que todos los estudios tenían el mismo tamaño del efecto poblacional, y que los tamaños de los efectos diferían sólo como resultado del error de muestreo. Sin embargo, éste no fue el caso y se utilizó una aproximación más general (modelo de efectos aleatorios). Este análisis fue conducido utilizando el programa 5.3 de metaanálisis ${ }^{5}$.

La Tabla 2 muestra los tamaños de los efectos de los estudios seleccionados. La correlación $r$ de tamaño de efecto fue calculada desde un valor de ji cuadrado de un grado de libertad, tomando la raíz cuadrada del valor de ji cuadrado dividida por el número de casos dentro de la categoría del orden de nacimiento dado. El estimador del tamaño del efecto d fue obtenido de la correlación del tamaño del efecto $r$ usando la fórmula $d=2 r / \sqrt{ }\left(1-r^{2}\right)$. El tamaño del efecto refleja la magnitud de la diferencia entre el grupo de casos y la población en el grupo de edad materna dado en términos estandarizados. Este coeficiente es independiente del tamaño de la muestra del estudio. Los tamaños de los efectos fueron combinados en un smodelo de efectos aleatorios ${ }^{\sharp}$, siempre buscando alcanzar la homogeneidad. Esta estrategia permitió la combinación de los estudios que presentaron una distribución similar de casos y población por grupos de edad materna basada en los indicadores de homogeneidad en la Tabla 3 (varianza observada, error de varianza, varianza de poblaciones, y cantidad de varianza explicada por error de muestreo).

Los cálculos de $\mathrm{N}$ de Fail-Safe $\mathrm{N}$ dan cuenta del problema del file-drawer, un sesgo de muestreo en metaanálisis que expone a una seria amenaza a la validez de los resultados. Se asume que un número desconocido de estudios con efectos de tamaño cero permanecen abandonados en file-drawers, ya sea porque nunca han sido enviados a publicación 0 porque han sido rechazados. El Fail-Safe N informa sobre el número de estudios de file-drawer que se requieren para reducir el promedio de la

Tabla 1. Referencias identificadas por el método de selección utilizado y número de estudios con resultados confirmados por odds ratios (con intervalos de confianza de $95 \%$ ) calculada por los autores del presente estudio para madres de 19 años de edad o menores y de 40 años de edad o mayores

\begin{tabular}{|lccl|}
\hline Defecto & $\begin{array}{c}\mathrm{N}^{\circ} \text { Poblaciones } \\
\text { estudiadas }\end{array}$ & $\begin{array}{c}\text { No Resultados } \\
\text { confirmados }\end{array}$ & \multicolumn{1}{c|}{ Referencias } \\
\hline Anencefalia & 20 & 13 & $6-13,17,20,21,35,37,45-50$ \\
Espina bífida & 11 & 5 & $6,7,8,9,10,11,12,13,22,51,52$ \\
DTNs & 21 & 9 & $6-13,22-24,33,36,38,53-58$ \\
\hline
\end{tabular}

Nota: Las referencias 24 y 50 reportaron separadamente resultados para dos poblaciones independientes.

Tabla 2. Efectos de tamaño en los estudios seleccionados

\begin{tabular}{|llcccc|}
\hline Defecto & $\begin{array}{l}\text { Referencia y ubicación del } \\
\text { estudio }\end{array}$ & \multicolumn{2}{c}{$\begin{array}{c}\text { Tamaño de efecto } \\
\text { Edad materna } \leq 19 \text { años }\end{array}$} & \multicolumn{2}{c|}{$\begin{array}{c}\text { Tamaño de efecto } \\
\text { Edad materna } \geq 40 \text { años }\end{array}$} \\
\hline Anencefalia & & $\mathrm{d}^{*}$ & $\mathrm{r}$ & $\mathrm{d}$ & $\mathrm{r}$ \\
& 6.Malpas (1937) - Inglaterra & 1,35 & 0,56 & 0,67 & 0,32 \\
& 17.Böök, Rayner (1950) - Suecia & 0,05 & 0,025 & 0,0 & 0,0 \\
& 45.Corbett (1953) - Inglaterra & 0,53 & 0,26 & 0,77 & 0,36 \\
& 46.Searle (1959) - Singapur & 0,08 & 0,04 & 1,46 & 0,59 \\
& 20.Frézal et al. (1964) - Francia & 1,62 & 0,63 & 0,3 & 0,15 \\
& 47.Colman, Stoller (1968) - Australia & 0,18 & 0,09 & 0,4 & 0,2 \\
& 7.Czeizel, Révész (1970) - Hungría & 0,08 & 0,04 & 1,0 & 0,45 \\
& 21.Fedrick (1970) - Inglaterra, Escocia y Gales & 1,0 & 0,45 & 1,0 & 0,46 \\
\hline
\end{tabular}




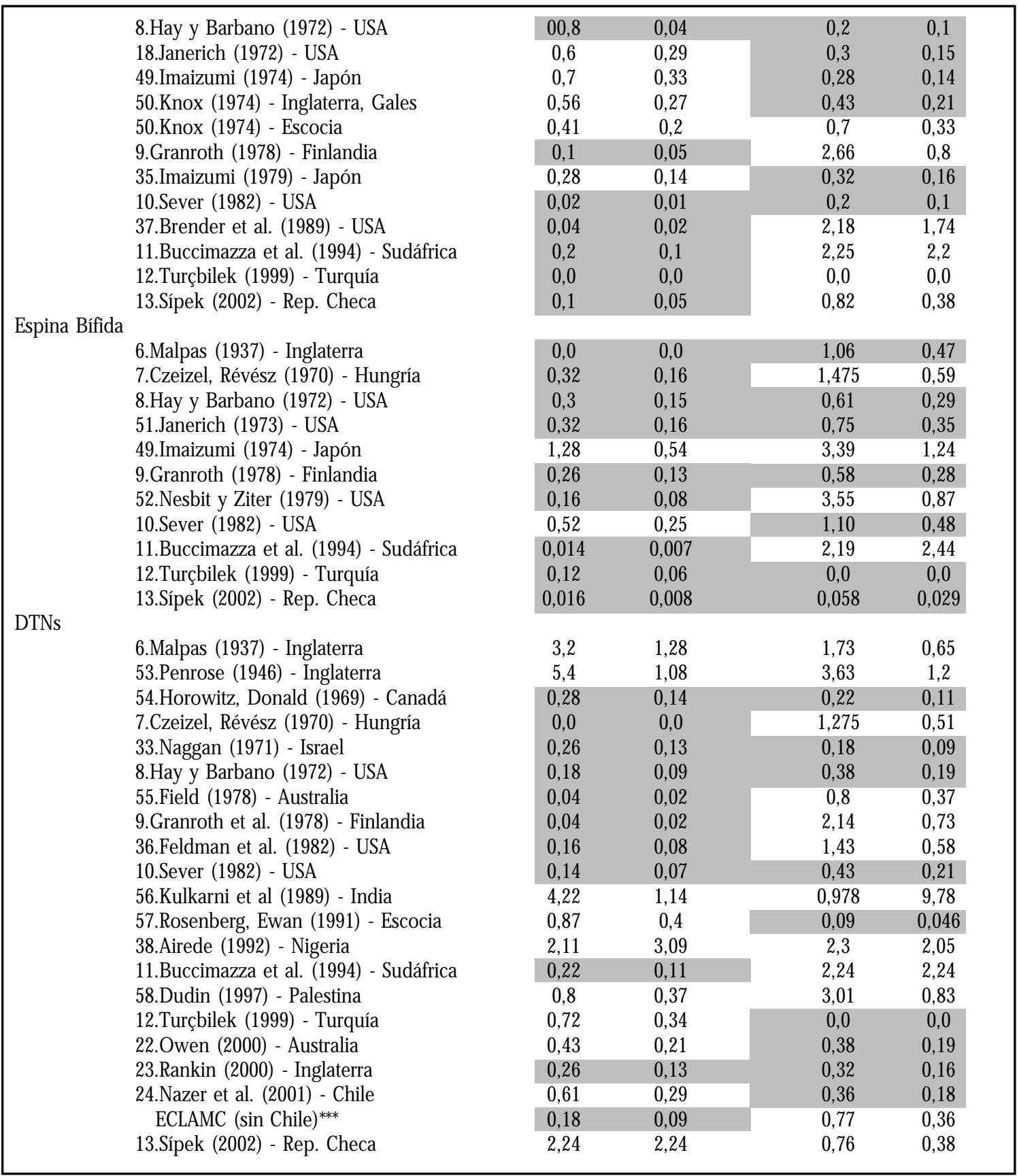

Notas: $d=$ estimador de tamaño de efecto * $*_{r}=$ correlación de tamaño de efecto

**ECLAMC es el Estudio Colaborativo Latino Americano de Malformaciones Congénitas y comprende información de hospitales en Argentina, Bolivia, Brasil, Chile, Colombia, Costa Rica, República Dominicana, Ecuador, Perú, Uruguay, Paraguay, y Venezuela.

Las zonas en gris corresponden a los estudios incluidos en el metaanálisis de tamaños de efecto combinados (Tabla 3). 
magnitud del efecto a un nivel definido. La fórmula para probabilidades de efecto de tamaño d es:

$\mathrm{N}_{\text {fail-safe }}=\left(\mathrm{N}_{\text {total }}\left(\right.\right.$ promedio Delta $\left.\left.-\mathrm{D}_{\text {crítico }}\right)\right) / \mathrm{D}_{\text {cńtico }}$

Los datos referentes a edad paterna, orden de nacimiento, nivel socioeconómico o etnicidad, por grupos de edad materna, no fueron incluidos en la mayoría de los estudios seleccionados y todos los análisis fueron conducidos sin ajuste alguno.

\section{Resultados}

De los 33 estudios seleccionados para análisis, 19 presentaban datos para anencefalia, 11 para espina bífida, y 20 para DTNs. La mayoría de las conclusiones originales están basadas en diferencias entre casos y frecuencias relativas en la población. En el presente trabajo, se calcularon los odds ratios para suministrar el nivel de significación de las diferencias reportadas. Si se considera la significación de odds ratio e intervalos de confianza sobre 1,0, numerosos resultados reportados no serían considerados significativos (Tabla 1).

La Tabla 2 muestra los resultados de los metaanálisis de la magnitud de efectos combinados. La cantidad de varianza explicada por error de muestreo en todas las comparaciones es $100 \%$, sugerente de que los datos fusionados son homogéneos. El delta promedio del efecto estimado para el grupo de edad materna «19 años» es pequeño para anencefalia $(0,08)$. Espina bífida presenta un delta promedio de efecto moderado para el grupo de edad materna «19 años» $(0,24)$, sugiriendo que hay un riesgo mayor de tener un niño con espina bífida en madres de 19 años de edad o menores. Parece que, el delta promedio del tamaño de efecto se diluye $(0,18)$ cuando se combinan los casos de anencefalia y espina bífida (grupo DTNs).

Para espina bífida y grupo de edad materna «19 años», entre uno y dos estudios de file drawer con tamaño de efecto de 0,0 son necesarios para reducir el delta de tamaño del efecto de población de 0,24 a un tamaño del efecto promedio de 0,20.

El efecto promedio estimado para el grupo de edad materna de « $₫ 40$ años» es moderado a alto en los tres grupos de enfermedad (0,37 para anencefalia, 0,65 para espina bífida, y 0,25 para DTN). También aparece que el delta promedio del efecto se diluye cuando los datos de anencefalia y espina bífida se combinan (grupo DTNs). Para DTNs, son necesarios entre dos y tres estudios de file drawer con tamaños de efectos 0,0 para reducir el delta del tamaño del efecto de la población de 0,25 a un tamaño de efecto promedio de 0,20. Entre seis y siete estudios con tamaños de efecto de 0,0 son necesarios para reducir el delta de tamaño de efecto de la población de 0,37 a un tamaño de efecto promedio de 0,20 para anencefalia, mientras que para espina bífida son necesarios entre trece y catorce estudios con tamaños de efectos 0,0 para reducir el delta de tamaño de efecto de la población de 0,65 a un tamaño de efecto promedio de 0,20.

Tabla 3. Resultados del metaanálisis para modelo de efectos aleatorios

\begin{tabular}{|c|c|c|c|c|c|c|}
\hline & \multicolumn{2}{|c|}{ Anencefalia } & \multicolumn{2}{|c|}{ Espina Bífida } & \multicolumn{2}{|c|}{ DTN } \\
\hline & $\begin{array}{l}\text { Edad matema } \\
\leq 19 \text { años }\end{array}$ & $\begin{array}{l}\text { Edad matema } \\
\geq 40 \text { años }\end{array}$ & $\begin{array}{l}\text { Edad matema } \\
\leq 19 \text { años }\end{array}$ & $\begin{array}{l}\text { Edad matema } \\
\geq 40 \text { años }\end{array}$ & $\begin{array}{l}\text { Edad matema } \\
\leq 19 \text { años }\end{array}$ & $\begin{array}{l}\text { Edad matema } \\
\geq 40 \text { años }\end{array}$ \\
\hline Número de sets de datos & 10 & 8 & 8 & 6 & 10 & 8 \\
\hline $\begin{array}{l}\text { Delta promedio de tamaño de } \\
\text { efecto (error standard) }\end{array}$ & $\begin{array}{l}0,08091 \\
(0,03485)\end{array}$ & $\begin{array}{l}0,37380 \\
(0,03937)\end{array}$ & $\begin{array}{l}0,24290 \\
(0,02332)\end{array}$ & $\begin{array}{l}0,65814 \\
(0,05483)\end{array}$ & $\begin{array}{l}0,18141 \\
(0,01795)\end{array}$ & $\begin{array}{l}0,25492 \\
(0,03622)\end{array}$ \\
\hline Intervalo de confianza 95\% & $\begin{array}{l}0,0126- \\
0,1492\end{array}$ & $\begin{array}{l}0,2966- \\
0,4510\end{array}$ & $\begin{array}{l}0,1972- \\
0,2886\end{array}$ & $\begin{array}{l}0,5507- \\
0,7656\end{array}$ & $\begin{array}{l}0,1462- \\
0,2166\end{array}$ & $\begin{array}{l}0,1839- \\
0,3259\end{array}$ \\
\hline Varianza observada & 0,00329 & 0,00680 & 0,01614 & 0,14506 & 0,00727 & 0,02125 \\
\hline Error de varianza & 0,06330 & 0,06300 & 0,07825 & 0,21427 & 0,01123 & 0,01406 \\
\hline Varianza de población & 0,00000 & 0,00000 & 0,00000 & 0,00000 & 0,00000 & 0,02897 \\
\hline $\begin{array}{l}\text { \% de varianza explicada por } \\
\text { error de muestreo }\end{array}$ & $100 \%$ & $100 \%$ & $100 \%$ & $100 \%$ & $100 \%$ & $100 \%$ \\
\hline Fail-Safe N & - & 6,952 & 1,716 & 13,7442 & & 2,1968 \\
\hline
\end{tabular}




\section{DisCUSIÓN}

El presente trabajo revisa la mayoría de los estudios que examinaron información relacionada a DTNs y edad materna. Mientras la anencefalia parece tener un riesgo aumentado para madres de 40 años de edad o mayores, espina bífida muestra una relación en forma de $\mathrm{U}$, con un mayor riesgo para madres de 19 años o menores y de 40 o más años. El efecto de la edad materna avanzada parece ser más intenso para espina bífida que para anencefalia. Los resultados discordantes que vemos en la literatura pueden ser explicados en parte por analizar los casos de anencefalia y espina bífida combinados, definidos como casos de defectos del tubo neural. Estudios que presenten mayores proporciones de casos de espina bífida tendrán mayor probabilidad de encontrar relaciones en forma de $\mathrm{U}$ o efectos más intensos en edad materna de 40 años o mayores. Estudios con mayor proporción de casos de anencefalia pueden tender a mostrar un efecto de edad materna de 40 años o más. Los resultados de los estudios realizados en la República Checa, Inglaterra, Finlandia, Hungría, Sudáfrica, Turquía y Estados Unidos de Norteamérica (USA) ${ }^{6-13}$ podrían estar reflejando las diferentes proporciones de anencefalia y espina bífida.

Se sabe que las madres de 40 años o mayores están en mayor riesgo para trisomía 13, 18, ó 21. La espina bífida no se reporta frecuentemente en síndrome de Down, pero está presente en menos de $50 \%$ de los casos de trisomía 13 y en menos de $10 \%$ de los casos de trisomía $18^{14}$. Los resultados del presente estudio pudieran estar afectados por diferencias en la forma de pesquisar los casos en los diversos trabajos. También debe considerarse que $15 \%$ de los pacientes con DTNs presenta alguna otra anomalía asociada o corresponde a algún síndrome ${ }^{15}$, y la inclusión de estos casos podría asimismo afectar los resultados.

Un metaanálisis tiene diversos sesgos potenciales, incluyendo sesgo de publicación, sesgo en la localización de los estudios (sesgo por idioma inglés, sesgo en las bases de datos, sesgo de citación, sesgo de múltiples publicaciones), sesgo en la provisión de datos, y pobre calidad metodológica ${ }^{16}$. Entre éstos, este estudio pudo haber eliminado sólo el sesgo de múltiples publicaciones. Esto revela la dificultad de la realización de este metaanálisis.
Los cálculos de odds ratio realizados en el presente trabajo no concuerdan con muchos resultados reportados (Tabla 1). La principal razón de ello es que, la mayoría de los resultados reportados estuvieron basados en comparaciones de frecuencias relativas de edades maternas entre casos y la población.

En este estudio, se utilizó una estimación del tamaño del efecto, porque las estimaciones del tamaño del efecto no están influenciadas por tamaños de la muestra. Hay una enorme variación en el tamaño muestral de los estudios seleccionados y las estadísticas tradicionales serían inapropiadas para las comparaciones realizadas en el presente estudio, porque los valores de aquellas estadísticas están parcialmente en función del tamaño muestral. Muchos estudios tienen un tamaño del efecto mayor a 0,2 (Tabla 2), lo que indica que la distribución del grupo de casos por edad materna difiere sustancialmente de la distribución observada en la población. Combinar todos los estudios, probablemente, arrojaría un resultado espúreo. Muchos hechos podrían contribuir a la diferencia observada entre la población y los casos en los estudios. Muy probablemente el factor más significativo sería la interrupción de los embarazos. Entre 1950 y 1985, la mayoría de los países desarrollados liberalizaron su legislación en torno al aborto, por razones de derechos humanos y seguridad. En India y Zambia, el aborto está legalmente permitido por razones socioeconómicas. Con la excepción de un estudio de anencefalia (17-Suecia), todos los estudios presentaron tamaños de efectos para anencefalia, espina bífida 0 DTNs sobre 0,2. La mayoría de los estudios seleccionados para el presente metaanálisis provienen de países donde la interrupción del embarazo está permitida, por ejemplo Inglaterra, Escocia, USA, Japón, Finlandia, Canadá, Hungría, Israel, Australia, e India (ver tabla 2 para las referencias). Los mayores efectos observados en los países donde el aborto es ilegal en todas las circunstancias, o permitido sólo para salvar la vida de la mujer o proteger su salud física (Nigeria y los países del estudio sudamericano), pueden ser debidos a otro factor. El estatus socioeconómico es el factor que podría justificar los mayores tamaños de los efectos, pero esta información no estaba disponible en éstos ni en la mayoría de los estudios incluidos en el presente metaanálisis. El nivel 
socioeconómico bajo, medido por ocupación y educación parental, ha sido encontrado asociado con un riesgo aumentado de tener un hijo con un DTN en algunos estudios ${ }^{18,19}$. Dificultades en la obtención de una medición consistente del nivel socioeconómico, especialmente a través de diferentes poblaciones y diversos países, pudiera explicar por qué esta variable no fue extensamente investigada. Los estudios que reportaron una asociación entre DTNs y madres más jóvenes pudieran estar reflejando un riesgo aumentado para nivel socioeconómico bajo ${ }^{20-24}$. Esto es consistente con los resultados del presente estudio. Este metaanálisis sugiere una asociación con madres de $\leq 19$ años de edad o menores sólo para espina bífida.

Otro importante factor, que ciertamente contribuyó a los diversos tamaños de los efectos vistos a través de los estudios presentados en la Tabla 2, es el trasfondo genético. La asociación más estrecha entre espina bífida y edad materna avanzada vista en este estudio, en contraste con la asociación débil vista para anencefalia, apoya la hipótesis de que estos dos defectos tienen algunos mecanismos etiológicos que son independientes. Los resultados para DTNs son probablemente guiados por la contrastante asociación que existe entre anencefalia y espina bífida y edad materna más avanzada, y por diferencias en la proporción de cada una de estas condiciones en el grupo total de los DTNs. Espina bífida y anencefalia, generalmente, se consideran una entidad porque ambos defectos pueden verse en las mismas familias ${ }^{25}$ y probablemente, esa es la razón de por qué tantos estudios agrupan ambos defectos. Es muy probable que espina bífida y anencefalia compartan algunos mecanismos, pero siempre debieran ser consideradas entidades independientes. Las diferencias en la razón de sexos apoyan esta aseveración. Hay un pequeño exceso de mujeres en el caso de espina bífida y un gran exceso con anencefalia. Por otro lado, este exceso de mujeres con anencefalia, no es visto en poblaciones de descendencia africana ${ }^{26}$. Esta también es evidencia de la importancia de ciertos factores genéticos. La variación geográfica vista para DTNs, también argumenta en favor de una fuerte contribución genética. El noroeste de las islas británicas tiene una de las tasas de prevalencia más altas registradas en el mundo, con aproximadamente $1 \%$ de los nacimientos afectados por anencefalia o espina bífida, mientras esta cifra es alrededor de cinco veces menor en la mayoría de las regiones sobre las cuales se han publicado datos confiables ${ }^{1}$.

Múltiples genes y factores ambientales han sido propuestos de estar involucrados en la etiología de DTNs. El factor de riesgo más significativo identificado en la actualidad es el ácido fólico ${ }^{27-29}$. Originalmente, se pensó que la deficiencia de folatos sería un representante del estado de bajo nivel socioeconómico, basado en los hallazgos en Gran Bretaña ${ }^{30,31}$. Sin embargo, Sudamérica, por ejemplo, tiene bajas tasas de prevalencia de anencefalia y espina bífida, a pesar de ser una región en vías de desarrollo, donde incluso se dan ingresos más bajos y dietas más pobres que aquellas de las clases sociales más bajas de Gran Bretaña ${ }^{32}$. Esta evidencia apoya la potencial importancia de los genes involucrados en el metabolismo de los folatos.

El orden de nacimiento y la edad paterna son otras variables que han sido reportadas como asociadas con DTNs. Un metaanálisis de la asociación entre los defectos del tubo neural y orden de nacimiento mostró que los niños con mayor orden de nacimiento, tienen más probabilidades de tener espina bífida, pero no anencefalia ${ }^{2}$. A pesar de que el orden de nacimiento y la edad materna no pudieron ser analizados en conjunto, muchos estudios sugieren un efecto independiente de estas dos variables ${ }^{24,33-39}$. La mayoría de los estudios no han demostrado una relación entre edad paterna y riesgo para $\mathrm{DTNs}^{7,18,35,40-44}$. No fue posible estudiar la edad paterna en el presente reporte, porque la mayoría de los estudios no incluían información respecto a la edad paterna.

En conclusión, un metaanálisis utilizando datos de treinta y tres estudios que investigaron una posible asociación entre edad materna y DTNs, sugiere que hay una asociación entre edad materna aumentada y ciertas formas de DTNs con un mayor efecto para espina bífida que para anencefalia. Espina bífida también parece estar asociada con madres de 19 años de edad o menores. Por ello, en la actualidad, en que sólo un pequeño porcentaje de defectos de nacimiento son prevenibles, el conocimiento de cualquier factor de riesgo que aumente el riesgo para un defecto común como DTNs es relevante. Una atención especial a la ingesta de ácido fólico y al cuidado preconcepcional, pueden proporcionar una protección adicional para al menos un porcentaje de todos los embarazos. 


\section{REFERENCIAS}

1. Elwood JM, ELwood JH. Epidemiology of anencephaly and spina bifida. New York: Oxford University Press. 1980.

2. VIEIRA AR. Birth order and neural tube defects: a reappraisal. J Neurol Sciences 2004; 217: 65-72.

3. Dean AG, Dean JA, Coulombier D Brendel KA, Smith DC, Burton AH, Dicker RC, SuLuvan K, Fagan RF, ARNer TG. Epi Info, Version 6: A WordProcessing, Database, and Statistics Program for Public Health on IBM-compatible Microcomputers. Centers for Disease Control and Prevention, Atlanta, Georgia, U.S.A. 1995.

4. Hedges LV, Olkin I. Statistical methods for metaanalysis. New York: Academic Press. 1985.

5. Schwarzer R. Meta-Analysis 5.3. Free University of Berlin, Berlin. 1989.

6. MaLPAS P. The incidence of human malformations and the significance of changes in the maternal environment in their causation. J Obstet Gynaecol Brit Emp 1937; 44: 434-54.

7. Czeizel A, RÉvész C. Major malformations of the central nervous system in Hungary. BritJ Prev Soc Med 1970; 24: 205-22.

8. HaY S, BaRBano H. Independent effects of matemal age and birth order on the incidence of selected congenital malformations. Teratology 1972; 6: 271-80.

9. Granroth G, HaApakoski J, Hakama M. Defects of the central nervous system in Finland. II. Birth order, outcome of previous pregnancies and family history. Teratology 1978; 17: 213-22.

10. SEver LE. An epidemiologic study of neural tube defects in Los Angeles County. II. Etiologic factors in an area with low prevalence birth. Teratology 1982; 25: 323-34.

11. Buccimazza SS, Molteno CD, Dunne TT, Viljoen DL Prevalence of neural tube defects in Cape Town, South Africa. Teratology 1994; 50: 194-9.

12. TunçBilek E, Boduroglu K, Alukasifoglu M. Neural tube defects in Turkey: prevalence, distribution and risk factors. Turk J Pediatr 1999; 41: 299-305.

13. Sípek A, Horácek J, Gregor V, Rychtaríková J, Dzurová D, Masátová D. Neural tube defects in the Czech Republic during 1961-1999: incidence, prenatal diagnosis and prevalences according to maternal age. J Obst Gynaecol 2002; 22: 501-7.

14. JoNES KL. Smith's recognizable patterns of human malformation. 5th rev. ed. Philadelphia: W.B. Saunders. 861p. 1997.
15. SELIER MJ. Sex, neural tube defects, and multisite closure of the human neural tube. Am J Med Genet 1995; 58: 332-6.

16. EgGeR M, SMIth GD. Meta-analysis: Bias in location and selection of studies. Brit Med J 1998; 316: 61-6.

17. BöÖK JA, RAYNER S. A clinical and genetical study of anencephaly. Am J Hum Genet 1950; 2: 61-84.

18. Strassburg MA, Greenland S, Portigal LD, Sever LE. A population-based case-control study of anencephalus and spina bifida in a low-risk area. Dev Med Child Neurol 1983; 25: 632-41.

19. Canfield MA, Annegers JF, Brender JD, Cooper SP, GREENBERG F. Hispanic origin and neural tube defects in Houston/Harris County, Texas (I \& II). Am J Epidemiol 1996; 143: 1-24.

20. Frézal J, Keuley J, Guilemot ML, Lamy M. Anencephaly in France. Am J Hum Genet 1964; 16: 336-50.

21. FEDRICK J. Anencephalus: variation with matemal age, parity, social class and region in England, Scotland and Wales. Ann Hum Genet 1970; 34: 31-8.

22. Owen TJ, HaLdday JL, Stone CA. Neural tube defects in Victoria, Australia: potential contributing factors and public health implications. Aust $\mathrm{N}$ Z J Public Health 2000; 24: 584-9.

23. Rankin J, Gunianaia S, Brown R, Renwick M. The changing prevalence of neural tube defects: a population-based study in the north of England, 1984-96. Paediatr Perin Epidemiol 2000; 14: 104-10.

24. Nazer JH, López-Camelo, CASTiLa EE. ECLAMC: estudio de 30 años de vigilancia epidemiológica de defectos de tubo neural en Chile y en Latinoamérica. Rev Méd Chile 2001; 129: 531-9.

25. Funrmann W, Seeger W, Bohm R. Apparently monogenic inheritance of anencephaly and spina bifida in a kindred. Humangenetik 1971; 13: 241-3.

26. CARTER CO. Spina bifida and anencephaly: a problem in genetic-environmental interaction. J Biosoc Sci 1969; 1: 71-83.

27. MRC Vitamin Study Research Group. Prevention of neural tube defects. Lancet 1991; 338:131-137.

28. Honein MA, Paulozzi LJ, Mathews TJ, ERickson JD, Wong LYC. Impact of folic acid fortification of the food supply on the occurrence of neural tube defects. J Am Med Assoc 2001; 285: 2981-6.

29. Castila EE, Oriol IM, Lopez-Camelo JS, Dutra Mda G, Nazer-Herrera J; Latin American Colaborative Study of Congenital Malformations (ECLAMC). Preliminary data on changes in neural tube defect prevalence rates after folic acid fortification in South America. Am J Med Genet 2003; 123A: 123-8. 
30. Smithells RW, Sheppard S, Schorah CJ, Seluer MJ, Nevin NC, HaRRis R et al. Possible prevention of neural tube defects by periconceptional vitamin supplementation. Lancet 1980; 1: 339-40.

31. Smithells RW, Nevin NC, Seluer MJ, Sheppard S, HaRRIS R, READ AP ET aL. Further experience of vitamin supplementation for preventation of neural tube defects recurrences. Lancet 1983; 1: 1027-31.

32. CAStiLA EE, Oriol IM. Epidemiology of neural tube defects in South America. Am J Med Genet 1985; 22: 695-702.

33. NAGGAN L. Anencephaly and spina bifida in Israel. Pediatrics 1971; 47: 577-86.

34. Elwood JM, McBRide ML. Contrasting effects of maternal fertility and birth rank on the occurrence of neural tube defects. Epidemiol Comm Health 1979; 33: 78-83.

35. Imaizum Y. Anencephaly in Japan: paternal age, maternal age and birth order. Ann Hum Genet 1979; 42: 445-55.

36. Feldman JG, Stein SC, Kiein RJ, Kohl S, Casey G. The prevalence of neural tube defects among ethnic groups in Brooklyn, New York. J Chron Dis 1982; 35: 53-60.

37. Brender JD, Carmichael L, Preece MJ, Larimer GC, SuAREZ L. Epidemiology of anencephaly in Texas, 1981-1986. Texas Med 1989; 85: 33-5.

38. AirEDE KI. Neural tube defects in the middle belt of Nigeria. J Trop Pediatr 1992; 38: 27-30.

39. Elwood M, LtTLE J. Maternal age and reproductive history. In: Elwood JM, Little J, Elwood JH. Epidemiology and control of neural tube defects. Oxford: Oxford University Press, 1992. p. 391414.

40. HunTER AG. Neural tube defects in Eastern Ontario and Western Quebec: demography and family data. Am J Med Genet 1984; 19: 45-63.

41. FRECKER M, FRASER FC. Epidemiological studies of neural tube defects in Newfoundland. Teratology 1987; 36: 355-61.

42. Simpson JL, Mils J, Rhoads GG, Cunningham GC, Conley MR, Hoffman HJ. Genetic heterogeneity in neural tube defects. Ann Genet 1991; 34: 27986.

43. Chatkupt S, Skurnick JH, Jaggi M. Mitruka K, KoENIGSBERGER MR, Johnson WG. Study of genetics, epidemiology, and vitamin usage in familial spina bifida in the United States in the 1990s. Neurology 1994; 44: 65-70.
44. Shaw GM, Jensvold NG, Wasserman CR, Lammer EJ. Epidemiologic characteristics of phenotypically distinct neural tube defects among 0.7 million California births, 1983-1987. Teratology 1994; 49: 143-9.

45. CoRBEtT HV. The aetiology of anenchephaly. J Obst Gynaecol Brit Emp 1953; 60: 907-12.

46. SEARLE AG. The incidence of anencephaly in a polytypic population. Ann Hum Genet 1959; 23: 279-88.

47. CoLmann RD, Stower A. The occurrence of anencephalus in the state of Victoria, Australia. J Ment Def Res 1968; 12: 22-35.

48. JANERICH DT. Anencephaly and maternal age. Am J Epidemiol 1972; 95: 319-26.

49. Imaizum Y. Statistical analysis on anencephaly, spina bifida and congenital hydrocephaly in Japan. Jinrui Idengaku Zasshi 1974; 19:115-35.

50. KNox EG. Twins and neural tube defects. Brit J Prev Soc Med 1974; 28: 73-80.

51. JANERICH DT. Maternal age and spina bifida: longitudinal versus cross-sectional analysis. Am J Epidemiol 1973; 96: 389-95.

52. NesBit DE, Ziter FA. Epidemiology of myelomeningocele in Utah. Develop Med Child Neurol 1979; 21: 754-7.

53. PenRose LS. Familial data on 144 cases of anencephaly, spina bifida and congenital hydrocephaly. Eugenics 1946; 13: 73-98.

54. Horowitz I, McDonaLD AD. Anencephaly and spina bifida in the province of Quebec. Canad Med Ass J 1969; 100: 748-55.

55. FIELD B. Neural tube defects in New South Wales, Australia. J Med Genet 1978; 15: 329-38.

56. KulKarNi ML, Mathew MA, REDdy V. The range of neural tube defects in southern India. Arch Dis Child 1989; 64: 201-4.

57. Rosenberg K, McEwan HP. Teenage pregnancy in Scotland: trends and risks. Scot Med J 1991; 36: 172-4.

58. Dudin A. Neural tube defect among Palestinians: a hospital-based study. Ann Trop Paediatr 1997; 17: $217-22$.

Agradecimientos:

Gracias a Jeff Murray por muchas discusiones provechosas y a Sandra Daack-Hirsch y Dana Collins por la ayuda con el manuscrito. También a Cathy Alden de The American Society of Human Genetics por el envío de un apartado de la referencia Böök and Rayner, 1950. 